\title{
Study of the Weber number impact on secondary breakup of droplets of coal water slurries
} containing petrochemicals

Minakov A.V. ${ }^{1,2 *}$, Shebeleva A.A. ${ }^{1}$, Strizhak P.A. ${ }^{3}$, Chernetsky M.Yu. ${ }^{1}$, Volkov R.S. ${ }^{3}$

${ }^{1}$ Kutateladze Institute of Thermophysics, Russian Academy of Sciences, 1, Academician Lavrentiev Avenue, 630090, Novosibirsk, Russia

${ }^{2}$ Siberian Federal University, 79/10 Svobodny Avenue, 660041, Krasnoyarsk, Russia

${ }^{3}$ National Research Tomsk Polytechnic University, 30, Lenin Avenue, Tomsk, 634050, Russia

*Corresponding author: tov-andrey@yandex.ru

\begin{abstract}
The research presents the numerical modeling findings of the secondary breakup of droplets of coal water slurries containing petrochemicals (CWSP). The modeling method of the secondary droplet breakup is based on the VOF method for interface resolution, LES model for describing turbulence, and the technology of adapted dynamic grids. We have established the droplet breakup modes depending on the Weber number varying from 7 to 212. We have also examined the structure of the flow behind droplets. We have tested the numerical method, and the results are in good agreement with the existing experiments.
\end{abstract}

Keywords: coal water slurry containing petrochemicals, droplet, secondary breakup, mathematical modeling, VOF, LES.

\section{Introduction}

Dispersed systems comprised of water, milled coal or combustible coal processing wastes, chemical additives and oil processing waste are quite a promising alternative fuel for energy generation. Such fuels are referred to as coal water slurries containing petrochemicals (CWSP) or composite liquid fuels (CLF) $[1,2]$. The successful development of an effective combustion method for these fuels will engage lowquality combustible fossils into the fuel balance and enable recycling of environmentally harmful waste.

The main method of slurry fuel combustion is its preliminary atomization in a combustion chamber. This appreciably expands the contact area of the fuel with the oxidizer and intensifies heat and mass exchange. The jet atomization is accompanied by its primary breakup and formation of liquid droplets that suffer deformation under aerodynamic force, which can lead to droplet breakup (secondary breakup). The primary task in the development of new combustion technologies is to establish the dependence of this process on fuel properties and conditions of fuel supply into the chamber. 
In [3], M. Kurmangaliev et al. were probably the first to do an experimental study of the secondary breakup of CWS droplets [3]. T. U. Yu, S.W. Kang et al. in [4] examined the CWS spray for a coaxial nozzle. They elaborated on the empirical correlation for determining the average size of a spray particle depending on various parameters. We should also mention Sitarski's research $[5,6]$ investigating the evaporation of CWS droplets under an intense heat flow.

The first researchers to experimentally study the breakup of separate CSW droplets in detail were Hui Zhao et al. [7]. They explored the morphology of droplets for different We and Oh numbers and determined the time dependence of a CWS droplet total breakup on the Oh number. Later, Tavangar S. et al. in [8] presented a numerical study of separate modes of a droplet breakup discussed in the experimental study [7]. They obtained a satisfactory agreement of calculations with the experimental data in terms of a droplet shape.

Further experimental studies $[9,10]$ were aimed at determining the impact of rheological properties of a CWS on the deformation and breakup of jets and single droplets of a given fuel. The experimental observation provided the basis for determining the boundaries of jet and droplet breakup modes depending on the Weber numbers. They were found to depend largely on the composition and viscoplastic properties of the slurry fuel under study. Despite the existing research in the field, by now, the atomization and breakup of such viscoplastic liquids as CWS and CWSP are still understudied, especially from the theoretical perspective and in terms of numerical modeling. The existing approaches to modeling the two-phase flows of CWSP require further improvement and verification, which is primarily explained by the yield stress value and high viscosity of CWSP; this considerably affects the jet breakup process.

The aim of the present research is to numerically investigate the modes of deformation and breakup of single CWSP droplets versus the Weber number.

\section{Mathematical model of droplet breakup}

Nowadays, the gas-droplet flows can be described using a wide variety of mathematical models (see [11-13]). They are distinguished by methods of gas and particle movement description, accepted assumptions and limitations of use. As a rule, a suitable model is chosen with due consideration of available information about the flow structure and the required description accuracy. The methods of solving problems with moving interfaces are reviewed most comprehensively in [12-16].

In terms of the grid being used, all the numerical algorithms of moving interface resolution can be classified into three large groups [13]: the Lagrangian, the Eulerian and meshless methods. Meshlessmethods [17] use only a surface grid or no computation grid at all.

In the Lagrangian algorithms [18], the calculation nodes and cells move together with a continuous medium. This approach describes the interface very precisely and monitors its complex boundary 
conditions, such as surface tension force. However, the Lagrangian approach requires recalculation of the grid at every time step, being very time-consuming. Moreover, since the shape of the interface and its trajectory are often very intricate, the Lagrangian methods might lead to a significant distortion of the computed cells causing considerable errors in the results.

The Eulerian methods [19] are usually attractive for researchers because an immovable computation grid (often orthogonal and uniform) is being used. In this group of methods, the interface movement is tracked using discrete Lagrangian or continuous Eulerian markers. In terms of interface tracking, the Eulerian methods can be divided into three large groups of algorithms [19]: discrete point markers, discrete or continuous surface markers and continuous volume markers.

As of today, the methods of continuous markers are the most common ones. Nowadays, the high efficacy and implementation simplicity of the volume-of-fluid method (VOF) [20] make it the most popular one among the algorithms of continuous volume markers. It has proven itself in the calculation of flows with a free surface [12, 21-22]. The idea of the method is that a fluid and a gas are regarded as a single two-component medium; the spatial distribution of phases within the calculation domain is determined by the special marker function $F(x, y, z, t)$. The volumetric fraction of the liquid phase in the calculation cell is as follows: $F(x, y, z, t)=0$ for an empty cell, $F(x, y, z, t)=1$ for a cell entirely filled with liquid, $0<F(x, y, z, t)<1$ for a cell with the interface passing through it.

Since the free surface moves along with the liquid, the free boundary movement in the space is tracked by solving the transfer equation of liquid phase volume fraction in the cell:

$$
\frac{\partial F}{\partial t}+u_{i} \frac{\partial}{\partial x_{i}} F=0
$$

Where $u_{i}$ is the velocity vector of the two-phase medium found from solving a simultaneous hydrodynamics equation consisting of a mass conservation equation and a continuity equation:

$$
\frac{\partial \rho}{\partial t}+\frac{\partial}{\partial x_{i}}\left(\rho u_{i}\right)=0
$$

and a motion equation or the law of momentum conservation:

$$
\frac{\partial}{\partial t}\left(\rho u_{i}\right)+\frac{\partial}{\partial x_{j}}\left(\rho u_{i} u_{j}\right)=\frac{\partial}{\partial x_{j}}\left(\mu \frac{\partial \sigma_{i j}}{\partial x_{j}}\right)-\frac{\partial p}{\partial x_{i}}-\frac{\partial \tau_{i j}}{\partial x_{j}}+F_{S}
$$

where $\sigma$ is the tensor of viscous stresses, $F_{S}$ is the vector of volume forces, $p$ is static pressure, $\rho$ is the density of a two-phase medium.

The components of the tensor of viscous stresses $\sigma_{i j}$ :

$$
\sigma_{i j} \equiv\left[\mu\left(\frac{\partial u_{i}}{\partial x_{j}}+\frac{\partial u_{j}}{\partial x_{i}}\right)\right]-\frac{2}{3} \mu \frac{\partial u_{i}}{\partial x_{i}} \delta_{i j}
$$


where $\mu$ is the dynamic viscosity of a two-phase medium.

The density and molecular viscosity of the two-component medium under study are determined through the volumetric fraction of the liquid in a cell using the mixture rule:

$$
\begin{aligned}
& \rho=\rho_{1} F+(1-F) \rho_{2}, \\
& \mu=\mu_{1} F+(1-F) \mu_{2},
\end{aligned}
$$

Where $\rho_{1}, \mu_{1}$ are the density and viscosity of the liquid that is defined below; $\rho_{2}, \mu_{2}$ are gas density and viscosity.

The values of density $\rho$ and viscosity $\mu$ appear in the motion equation and determine the physical properties of the two-phase medium.

Since CWSs can exhibit non-Newtonian properties in certain cases, the non-Newtonian flows were modeled using the approach from [23-24], where a medium is considered a non-linear viscous liquid with the introduction of effective viscosity $\mu_{1}(\dot{\gamma})$, which normally depends on the shear rate.

The shear rate is, in general terms, the second invariant of the tensor of deformation rates:

$$
\dot{\gamma}=\sqrt{\frac{1}{2} \mathbf{D} \cdot \mathbf{D}}
$$

The components of the tensor of deformation rates $\mathbf{D}$ are as follows:

$$
\mathbf{D}_{i j}=\frac{\partial u_{i}}{\partial x_{j}}+\frac{\partial u_{j}}{\partial x_{i}}
$$

Thus, within the given model, a CWSP can be both a viscous Newtonian liquid and a non-Newtonian viscoplastic liquid whose behavior can be described by one of the four most commonly used rheological models: Power Law, Bingham-plastic and Herschel-Bulkley (power law Bingham fluid). Depending on the CWS rheology, the effective viscosity has the following form:

$$
\begin{aligned}
& \mu_{1}(\dot{\gamma})=k, \text { for Newtonian medium, where } k \text { is molecular viscosity of the liquid. } \\
& \mu_{1}(\dot{\gamma})=k \dot{\gamma}^{n-1} \text { for Power Law, } \\
& \mu_{1}(\dot{\gamma})=\frac{k \dot{\gamma}+\tau_{0}}{\dot{\gamma}} \text { for Bingham Plastic, } \\
& \mu_{1}(\dot{\gamma})=\frac{k \dot{\gamma}^{n}+\boldsymbol{\tau}_{0}}{\dot{\gamma}} \text { for Herschel-Bulkley, }
\end{aligned}
$$

where $n$ and $k$ are coefficients of rheological models, $\boldsymbol{\tau}_{0}$ is the yield point of a viscoplastic liquid (below a certain limit stress value, a medium behaves as a solid body, while above that, it behaves as an incompressible viscous liquid).

The consideration of liquid flows with an interface draws special attention to the surface tension. The study of flows controlled by surface tension forces is a really sophisticated problem by itself. In this 
connection, the strong points of the VOF method also include its ability to quite effortlessly simulate the effect of surface tension forces.

Most frequently, the surface tension forces within the VOF method are modeled by CSF [25] algorithm which implies the introduction of the additional volume force $F_{S}$ into the motion equation; its value is determined from the following ratio:

$$
F_{S}=\sigma k \nabla F,
$$

where $\sigma$ is a surface tension coefficient, $k$ is the curvature of the free surface determined as divergence of the normal vector:

$$
k=\nabla\left(\frac{n}{|n|}\right),
$$

In its turn, the normal to the free surface is calculated as a gradient of the volume fraction of the liquid phase in the cell:

$$
n=\nabla F
$$

The value of the normal vector on the solid wall is determined from the contact angle $\theta$ :

$$
n=n_{w} \cos \theta+\tau_{w} \sin \theta
$$

where $n_{w}, \tau_{w}$ are normal and tangential vectors to the wall.

Another important factor in the droplet breakup calculation is turbulence modeling. The present work uses LES model [26] to model the turbulence. Tensor $\tau_{i j}$ is referred to as the tensor of subgrid stresses; its components are determined similarly to RANS models from Boussinesq equation.

$$
\tau_{i j}-\frac{1}{3} \tau_{k k} \delta_{i j}=-2 \mu_{t} D_{i j}
$$

The component is referred to as subgrid viscosity. The present work uses the model of subgrid viscosity suggested by Smagorinsky:

$$
\mu_{t}=\rho L_{S}^{2} \dot{\gamma}
$$

The mixing length of subgrid scales is determined as:

$$
L_{S}=\min \left(k d, C_{S} V^{\frac{1}{3}}\right)
$$

Here, $k$ is von Kármán's constant, $d$ is the distance to the nearest wall, $V$ is the volume of the computed cell, $C_{S}$ is Smagorinsky constant. We used the value of $C_{S}=0.17$.

The method of solving equations (1)-(13) and the main peculiarities of numerical studies are described elsewhere [12, 27-28]. The difference analog of convective-diffusion equations was derived by means of the finite volume method for structured multi-block grids. It ensured the persistence of the elaborated scheme. To approximate the convective members of hydrodynamics equations (3), a central difference scheme of the second order was used. To approximate the nonstationary summands of hydrodynamics 
equations, an implicit scheme of the first order was used. The diffusion flows and source members were approximated with the second-order accuracy. The velocity and pressure fields were coupled by SIMPLEC procedures using combined grids. This approach allowed surpassing the above mentioned impediments connected with a moving interface resolution. The obtained system of difference equations was solved by the iteration method involving a multi-grid solver.

\section{Numerical simulation scheme}

To model the droplet breakup, we used a setup conventional for such cases. The calculation domain is a parallelepiped with the dimensions of $2.5 \times 2.5 \times 7 \mathrm{~cm}$. For one of the parallelepiped faces an entry condition was set with a specified velocity value determined from the Weber number. For the remaining faces of the calculation domain, the conditions of free exit were stated. At the initial moment of time, a spherical CWSP droplet with diameter $\mathrm{D}_{0}$ was placed at a distance of $5 \mathrm{~mm}$ from the entry into the calculation domain.

We used the uniform Cartesian calculation grid for the calculation. The total specification of the initial uniform grid comprised 8 million nodes. However, the systematic calculations have demonstrated that this grid is not sufficient for the resolution of the interface of forming small droplets. Therefore, we used gradient adaptation of the computation grid. This technology enables the grid to be automatically concentrated in the region of large solution gradients during the calculation. In this case, the gradient of the liquid volume fraction was chosen as the control parameter. A change in the initial grid during gradient adaptation for the flow velocity of $25 \mathrm{~m} / \mathrm{s}$ is exemplified in Fig. 1. The cells in the large gradient region are four times as small as those in the initial grid. As a result, the total number of calculation nodes in the optimized grid during the calculation was close to 23 million. The implementation of such a highly detailed grid allowed resolving secondary droplets with the dimensions down to $15 \mu \mathrm{m}$.

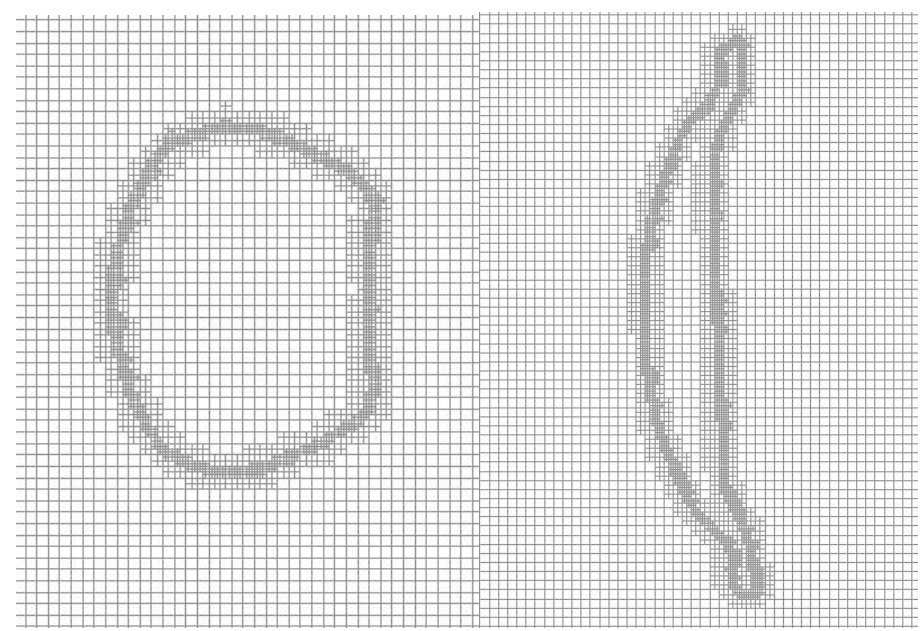

a)

b)

Fig. 1. Evolution of the computation grid with gradient adaptation during droplet deformation. 


\section{Testing of model and comparison with experimental data}

At the beginning of the study, we tested and verified the implemented numerical method of droplet breakup. To test the calculation method, a problem of CWS droplet breakup was considered. The problem was experimentally studied in [7]. The experimental setup and liquid properties in that work are close to the presently discussed problem of CWSP droplet breakup. The parameters of cases covered in the calculation are presented in Table 1.

Table 1. The parameters of cases

\begin{tabular}{|c|c|c|c|c|c|c|c|}
\hline Case & $\mathrm{D}_{0}[\mathrm{~mm}]$ & $\rho\left[\mathrm{kg} / \mathrm{m}^{3}\right]$ & $\tau_{0}[\mathrm{~Pa}]$ & $k\left[\mathrm{~Pa}{ }^{2}\right]$ & $\sigma[\mathrm{N} / \mathrm{m}]$ & $\mathrm{V}[\mathrm{m} / \mathrm{s}]$ & $\mathrm{We}$ \\
\hline 1 & 4.6 & 1179 & 1.96 & 0.06 & 0.0613 & 19.7 & 36 \\
\hline 2 & 4.8 & 1111 & 2.12 & 0.14 & 0.066 & 23.2 & 48 \\
\hline 3 & 4.8 & 1111 & 2.12 & 0.14 & 0.066 & 61.9 & 342 \\
\hline
\end{tabular}

Fig. 2 presents the behavior of a CWS droplet under breakup for cases 1-3. The first breakup mode ( $\mathrm{We}=36$ and $\mathrm{We}=48$ ) is characterized at the initial breakup stage by the behavior similar to the parachute breakup mode. The droplet swells into a shell. However, the flow then tears off a thin film from it, and the droplet turns into a ring, which further breaks up into separate fragments. Other researchers refer to such mode as a hole breakup. We can note that at $\mathrm{We}=36$ after the disruption of the liquid shell, a ring is formed, while in the center a relatively large fragment of the droplet remains with the dimensions of up to $20 \%$ of the initial droplet size. This fragment then decays rapidly.

The second mode $(\mathrm{We}=342)$ actually represents the catastrophic droplet breakup. In this mode, the droplet rapidly turns into a disc; however, unlike in the previous case, this disc does not swell in the direction of the flow, and the gas flow continuously blows off droplets from its external edge. As a result, the droplet very quickly turns into spray. 


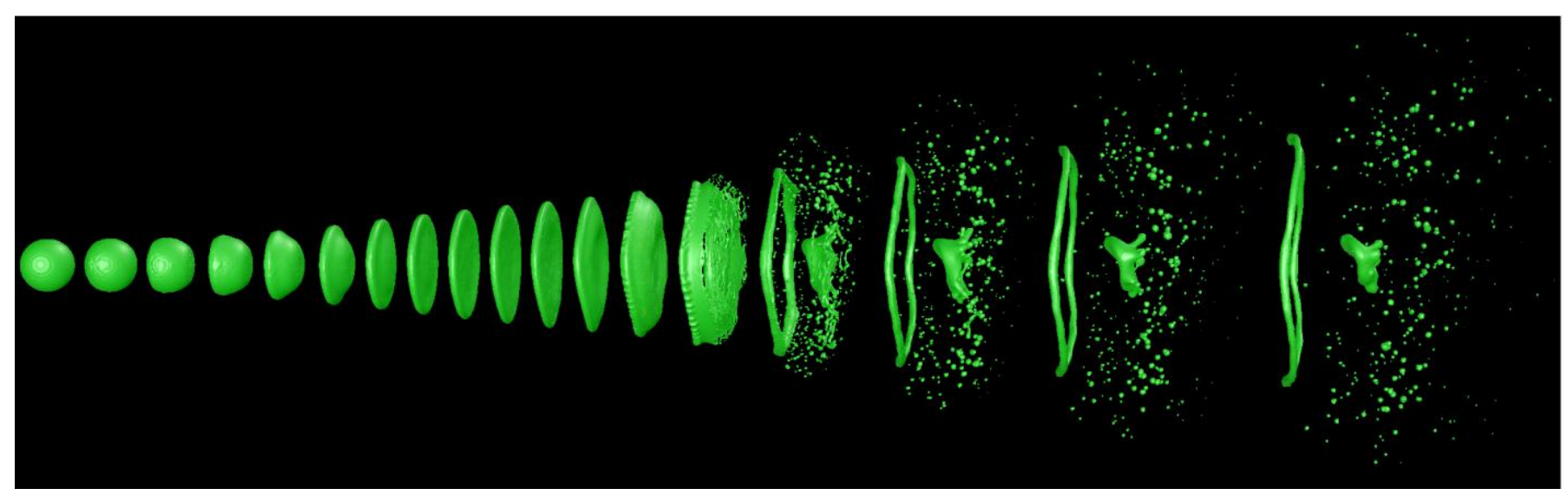

\section{$\mathrm{We}=36$}

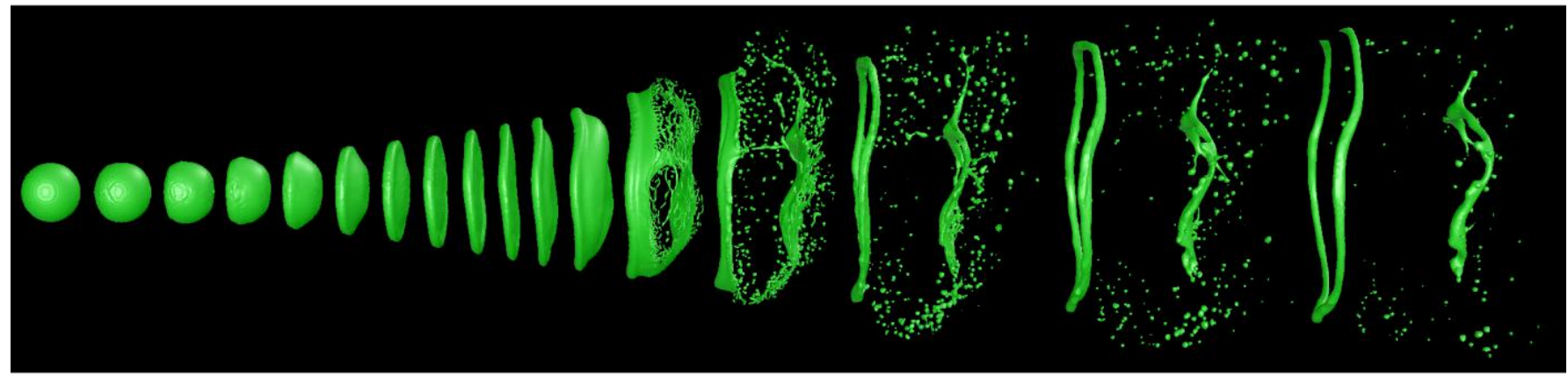

$\mathrm{We}=48$

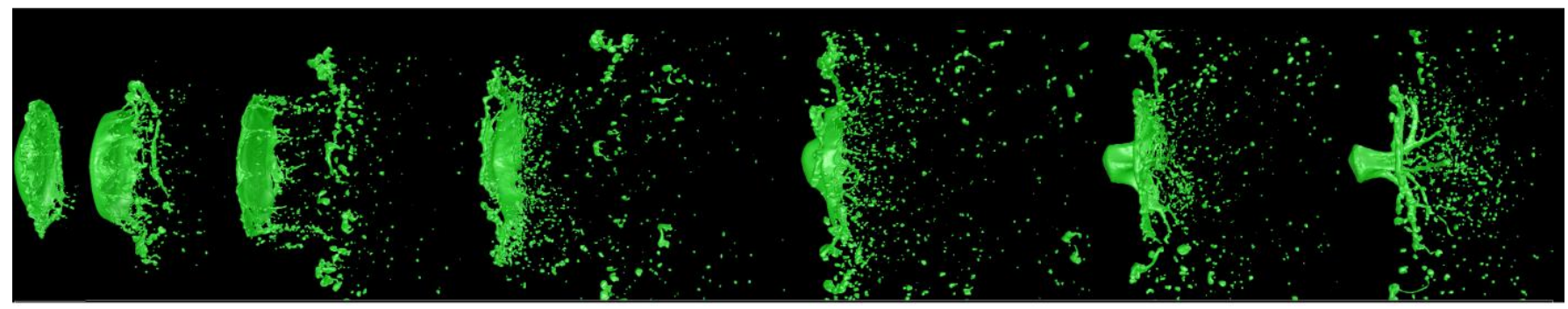

$\mathrm{We}=342$

Fig. 2. Dynamics of CWS droplet breakup for different modes.

The calculation and the experimental data on the dynamics of a CWS droplet breakup for the considered breakup modes are compared in Figs. 3-5. The analysis of the modeling results shows that for both of the modes under study the calculation satisfactorily describes the experimental data in terms of both the breakup process itself and the shape of droplet surface and their separate fragments at different moments of time. 

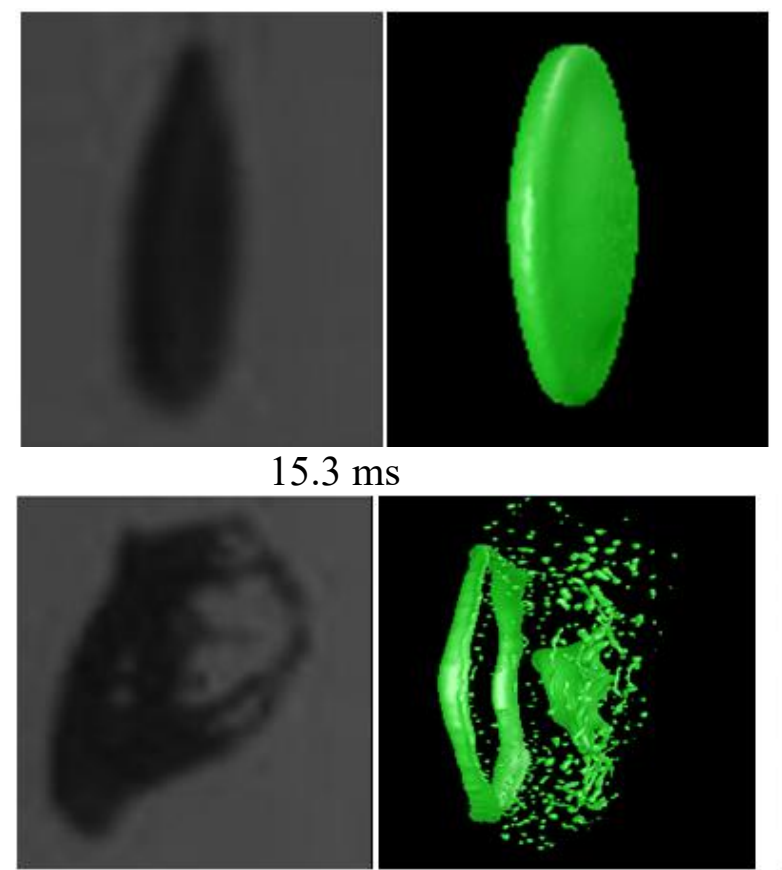

$17.3 \mathrm{~ms}$

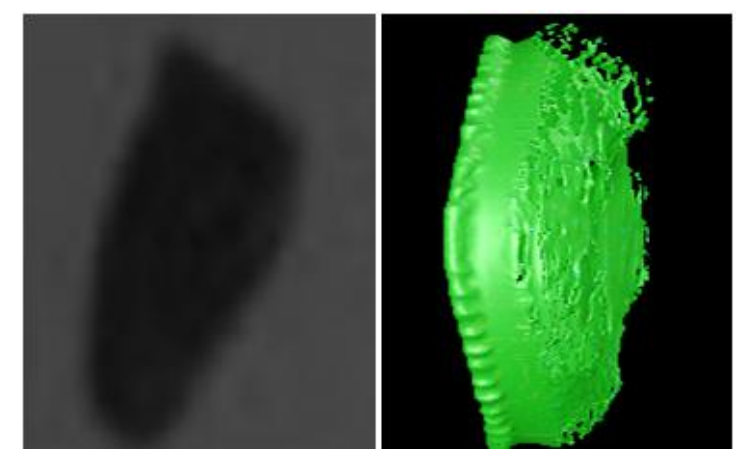

$16.3 \mathrm{~ms}$

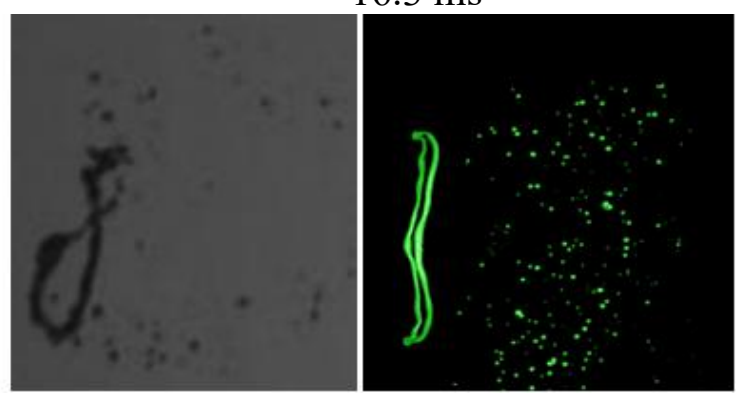

$22.3 \mathrm{~ms}$

Fig. 3. Comparison of experimental [7] (left-hand side) and calculated shape of CWS droplet surface at different moments of time at the flow velocity of $19.7 \mathrm{~m} / \mathrm{s}$.

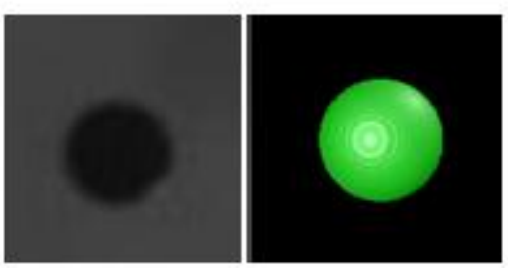

$0 \mathrm{~ms}$

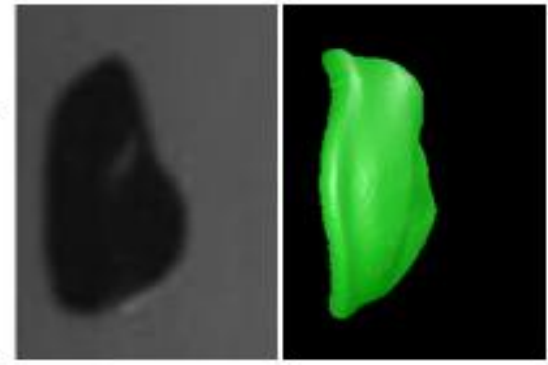

$10.7 \mathrm{~ms}$

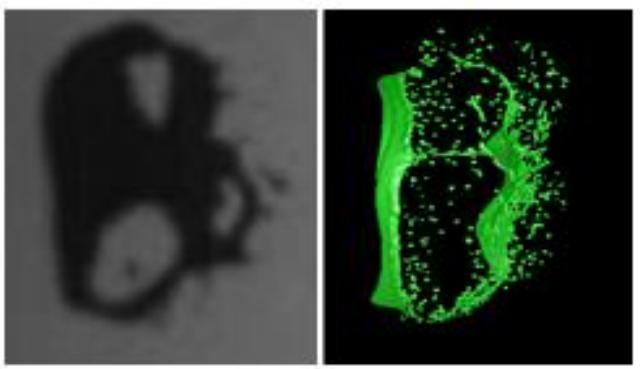

$12.7 \mathrm{~ms}$

Fig. 4. Comparison of experimental [7] (left-hand side) and calculated shape of CWS droplet surface at different moments of time at the flow velocity of $23.2 \mathrm{~m} / \mathrm{s}$.

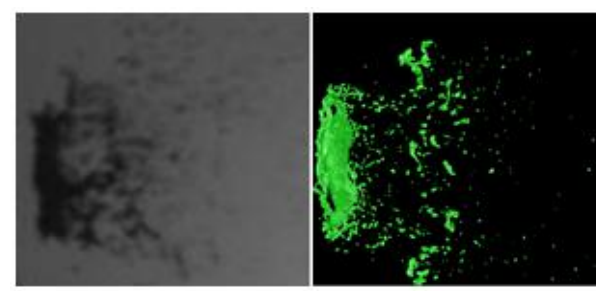

$3.7 \mathrm{~ms}$

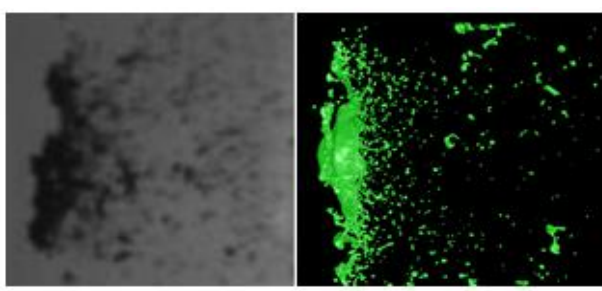

$4.7 \mathrm{~ms}$

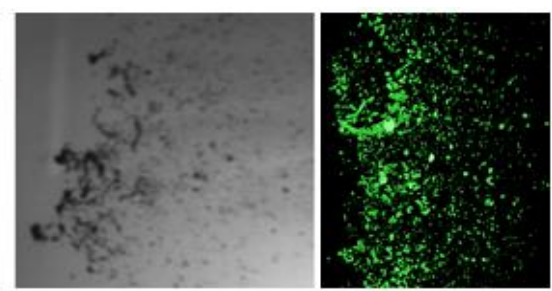

$7 \mathrm{~ms}$

Fig. 5. Comparison of experimental [7] (left-hand side) and calculated shape of CWS droplet surface at different moments of time at the flow velocity of $61.9 \mathrm{~m} / \mathrm{s}$.

\section{Results and discussion}

The above described method was used to model secondary breakup of a CWSP droplet with the filter cake content of $50 \%$ for different Weber number values. A filter cake is a waste product of coal flotation, which involves coal washing with a surfactant solution and further screening into separate fractions. Water used for coal washing is fed to special containers for clarification, where coal particles settle. This 
solution (slurry) is pumped and sent to belt press filters, where it is filtered through a special membrane and thus dewatered. The resulting wet residue of coal particles is a filter cake. In our research, we used filter cakes of coking coal produced with the addition of plasticizers whose properties conform well with Neolas stabilizer used in the experiments of [31].

The droplet diameter was $\mathrm{D}_{0}=5 \mathrm{~mm}$. The physical properties of a CWSP were specified according to the data from Table 1. The surface tension coefficient $\sigma=0.072 \mathrm{~N} / \mathrm{m}$. The viscosity of the liquid was measured using DV2T Rotational Viscometer (Brookfield, USA). The measurement results have shown that the rheology of the slurry under study is well described by the Bingham model $\mu_{1}(\dot{\gamma})=\frac{k \dot{\gamma}+\tau_{0}}{\dot{\gamma}}$, whose parameters are presented in Table 2.

Table 2. Main properties of CWSPs

\begin{tabular}{|l|c|c|c|c|}
\hline $\begin{array}{l}\text { Concentration of CWSP components } \\
\text { (relative mass fraction of initial wet } \\
\text { filter cake C in the composition) }\end{array}$ & $\begin{array}{c}\text { Density at } \\
293 \mathrm{~K}\left[\mathrm{~kg} / \mathrm{m}^{3}\right]\end{array}$ & $\begin{array}{c}\text { Moisture } \\
\text { content }[\%]\end{array}$ & $\tau_{0}[\mathrm{~Pa}]$ & $k\left[\mathrm{~Pa}^{*} \mathrm{~s}\right]$ \\
\hline Filter cake C $(50 \%)$ & 1115 & 71.75 & 3.9 & 0.006 \\
\hline
\end{tabular}

The secondary breakup of droplets of homogeneous liquids occurs under the aerodynamic force exceeding the surface tension forces. Quantitatively, the correlation of these forces is determined by the value of the deformation criterion, i.e. the Weber number $W e$ :

$$
W e=\frac{\rho_{g} U_{g}^{2} D_{0}}{\sigma},
$$

where $D_{0}=5 \mathrm{~mm}$ is the droplet diameter, $\sigma$ is the liquid surface tension coefficient, $\rho_{g}$ is the density of the surrounding gas, $U_{g}$ is gas velocity.

The value of the Weber number determines the mode of droplet breakup. To determine these modes, a series of calculations for different Weber numbers was performed. The flow velocity varied in the range from $9 \mathrm{~m} / \mathrm{s}$ to $50 \mathrm{~m} / \mathrm{s}$.

Fig. 6 shows the CWSP droplet behavior at the flow velocity of $9 \mathrm{~m} / \mathrm{s}$. This corresponds to the Weber number equal to 7. Obviously, at this Weber number value, there is no secondary breakup of a droplet. The movement of a droplet in the flow is accompanied by periodical oscillations of its shape. These oscillations are attenuating. The droplet oscillation period at the initial stage is close to $0.005 \mathrm{~s}$. The character of the flow behind a droplet for this mode is shown in Fig. 7. The flow around the droplet is accompanied by the formation of a sophisticated vortex-like structure behind it, which becomes most intense in the moments the droplet turns into a disk. When the shape of the droplet is close to a sphere, its flow-around coincides with the character of the flow in the case of the flow-around of a sphere, when a Karman vortex street is formed behind the droplet. This can be clearly seen in Fig. 7. 


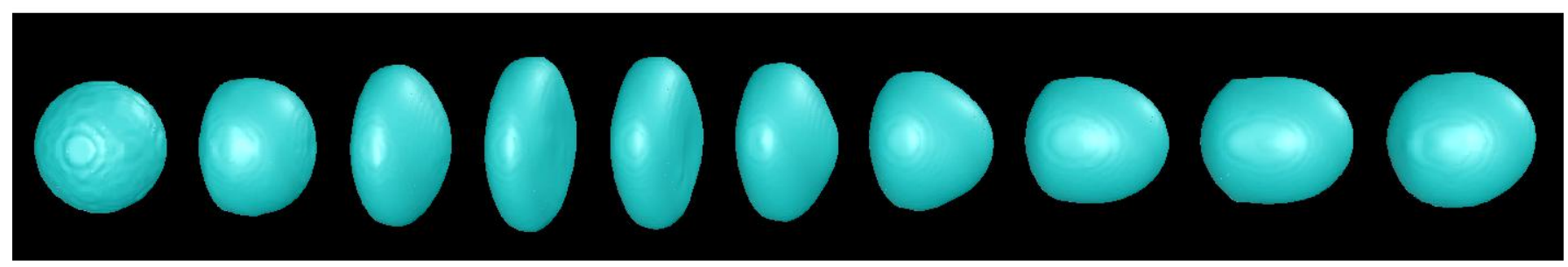

Fig. 6. Shape of droplet surface at different moments of time at the flow velocity of $9 \mathrm{~m} / \mathrm{s}$. The interval between frames is $4 \mathrm{~ms}$.

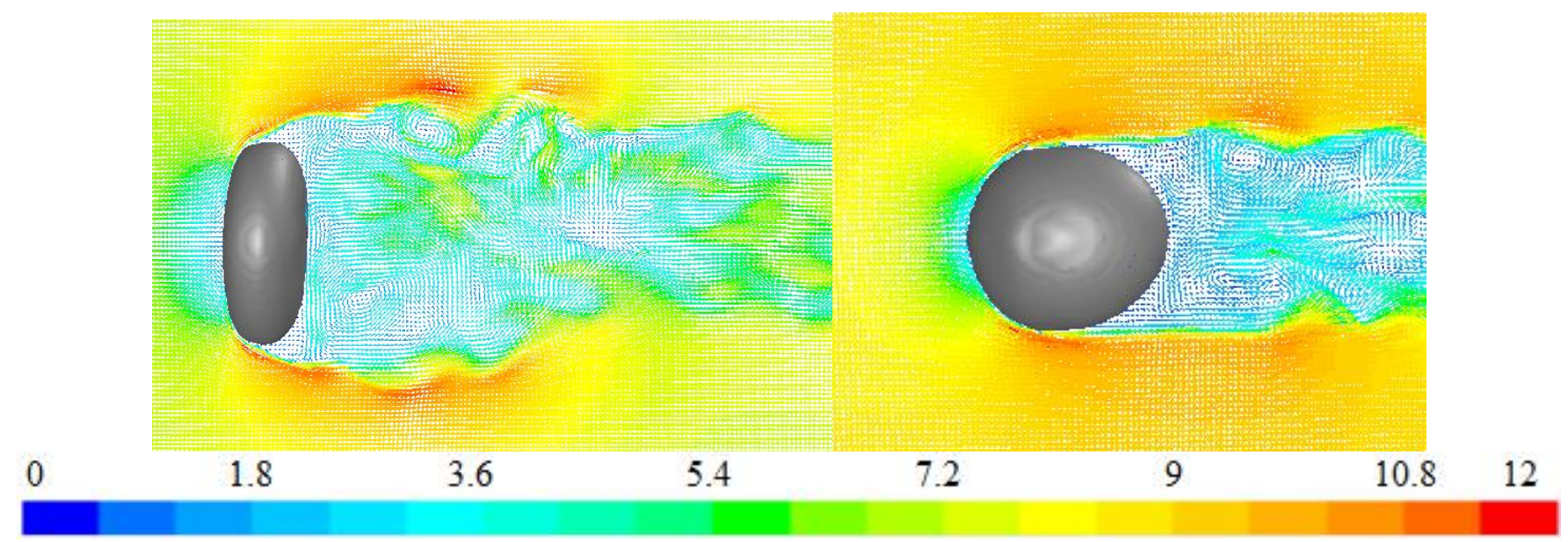

Fig. 7.Snapshot of the flow at different time moments at droplet flow-around at the flow velocity of $9 \mathrm{~m} / \mathrm{s}$.

Fig. 8 displays the droplet behavior evolution at the flow velocity of $12 \mathrm{~m} / \mathrm{s}$. This corresponds to the Weber number equal to 12.5. Evidently, the droplet behavior at this Weber number differs greatly from the above mentioned case. First, the droplet is deformed by the flow into a thin disk. Then, similarly to a soap bubble or a parachute, the droplet swells into liquid film. This is the so-called bag breakup or parachute mode of droplet breakup. The film of the liquid is rapidly thinned and soon tears down forming a plethora of small droplets and a liquid ring. Subsequently, the liquid ring breaks, too, and larger droplets are formed.

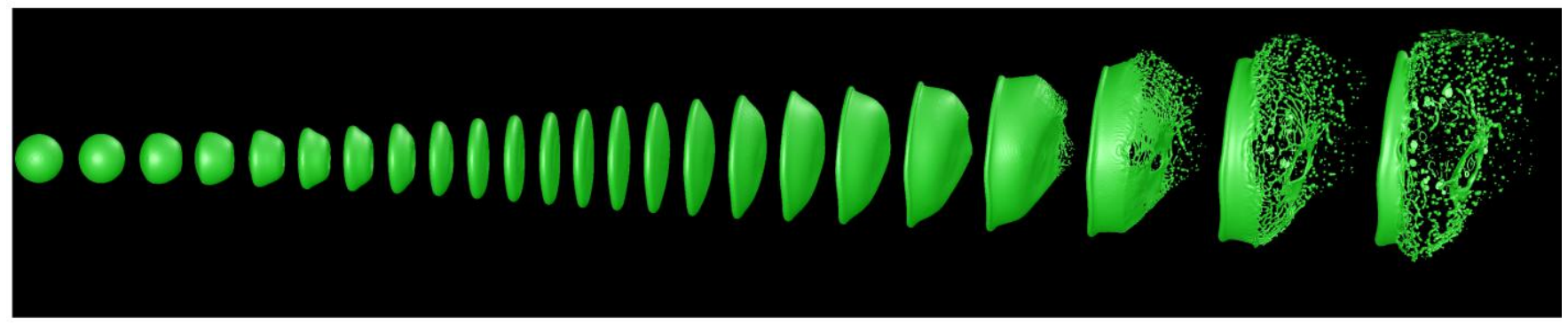

Fig. 8. Shape of droplet surface at different time moments at the flow velocity of $12 \mathrm{~m} / \mathrm{s}$. The interval between frames is $5 \mathrm{~ms}$.

With further increase in the flow velocity, one more interesting breakup mode is revealed which is shown in Fig. 9. This mode corresponds to the flow velocity of $15 \mathrm{~m} / \mathrm{s}$ and the Weber number equal to 
19.6. This is the so-called multi-model breakup or mixed breakup mode. At the beginning, it is very similar to the parachute breakup mode. A droplet is deformed into a thin disk which swells into a bubble. However, after the liquid film tears down, a jet directed against the flow is formed in its center.

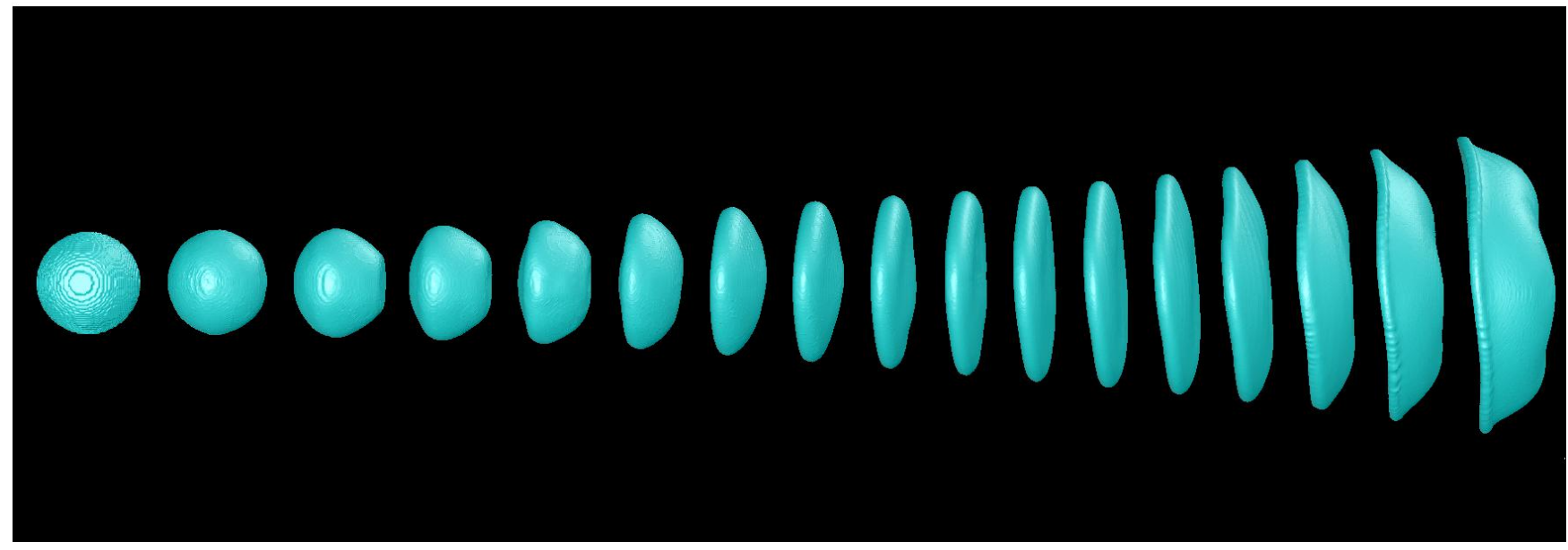

The interval between frames is $5 \mathrm{~ms}$.

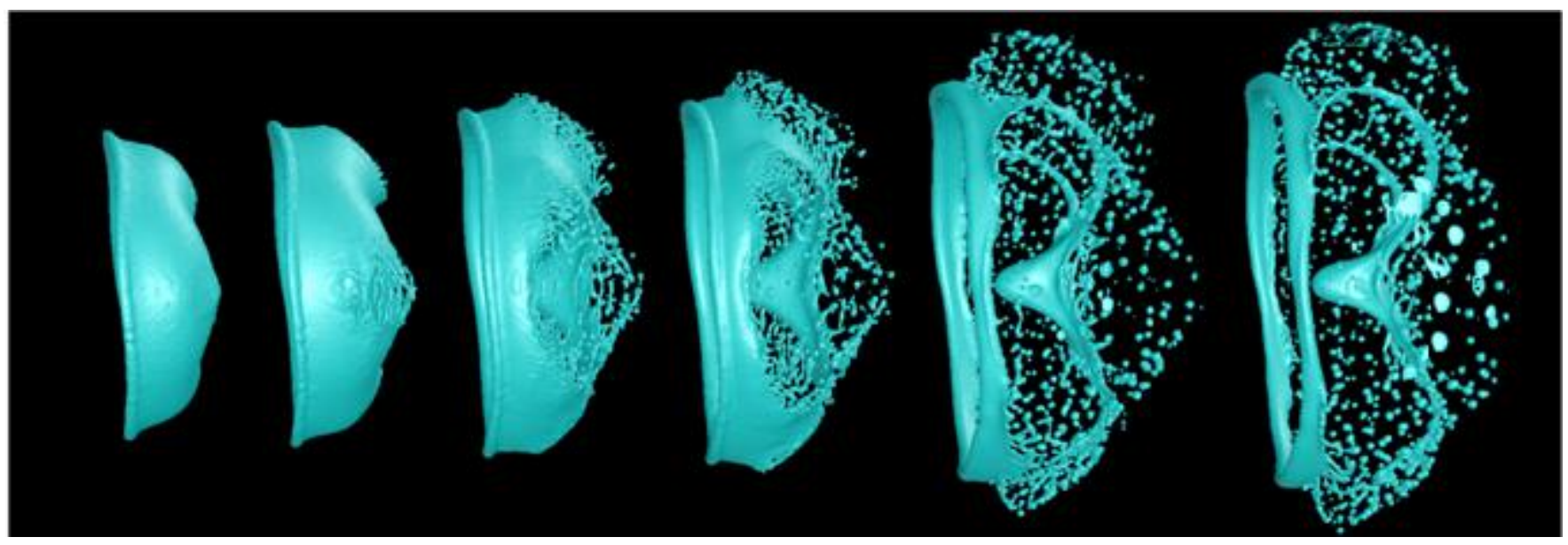

The interval between frames is $2.5 \mathrm{~ms}$.

Fig. 9. Shape of droplet surface at different moments of time at the flow velocity of $15 \mathrm{~m} / \mathrm{s}$.

At the flow speed of $20 \mathrm{~m} / \mathrm{s}$ (We=35), the breakup mode changes considerably. The evolution of the droplet at this speed is depicted in Fig. 10. Evidently, unlike previous parachute modes, the swelling of the liquid film occurs in the counterflow. That is why a relatively large droplet is formed in the center of the liquid film and moves in the opposite direction. From this droplet, several rather extended liquid jets are stretched in the direction of the gas flow. Further, all these structures break up into smaller droplets. 


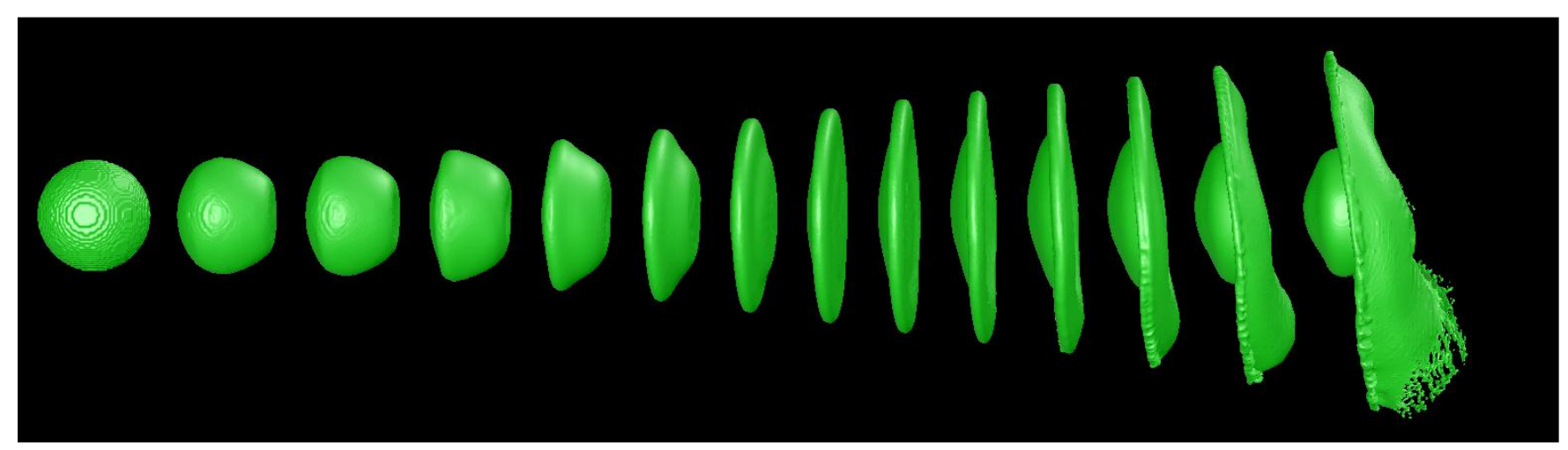

The interval between frames is $1 \mathrm{~ms}$.

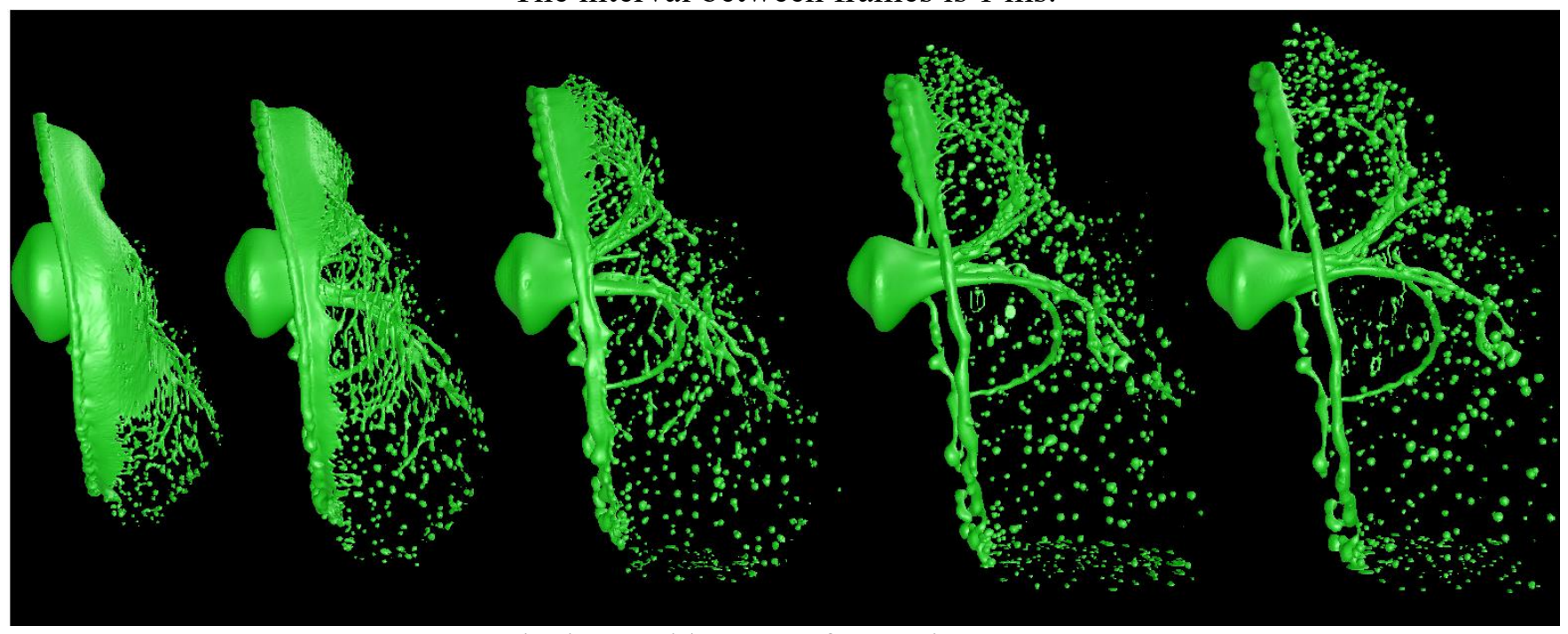

The interval between frames is $0.5 \mathrm{~ms}$.

Fig. 10.Shape of droplet surface at different moments of time at the flow speed of $20 \mathrm{~m} / \mathrm{s}$.

An interesting breakup mode, which is shown in Fig. 11, is formed at the flow speed of $25 \mathrm{~m} / \mathrm{s}$ $(\mathrm{We}=54)$. At the initial stage of evolution - similarly to the previous mode - the droplet swells in the counterflow. However, the liquid film does not break into small droplets. Instead, the flow tears off a ring from the outside edge of the liquid film. Then, this ring is rapidly carried downstream and torn into separate pieces. In the center, a liquid disk remains with jets stretching from it along the flow. The behavior of instantaneous velocity field at different moments of time for this mode is shown in Fig. 12 . Evidently, the velocity field behind the droplet is very intricate. The flow is primarily three-dimensional and non-stationary and depends on the droplet shape at the current moment of time. The implementation of LES method enables the resolution of a large-scale turbulent structure of the flow. 


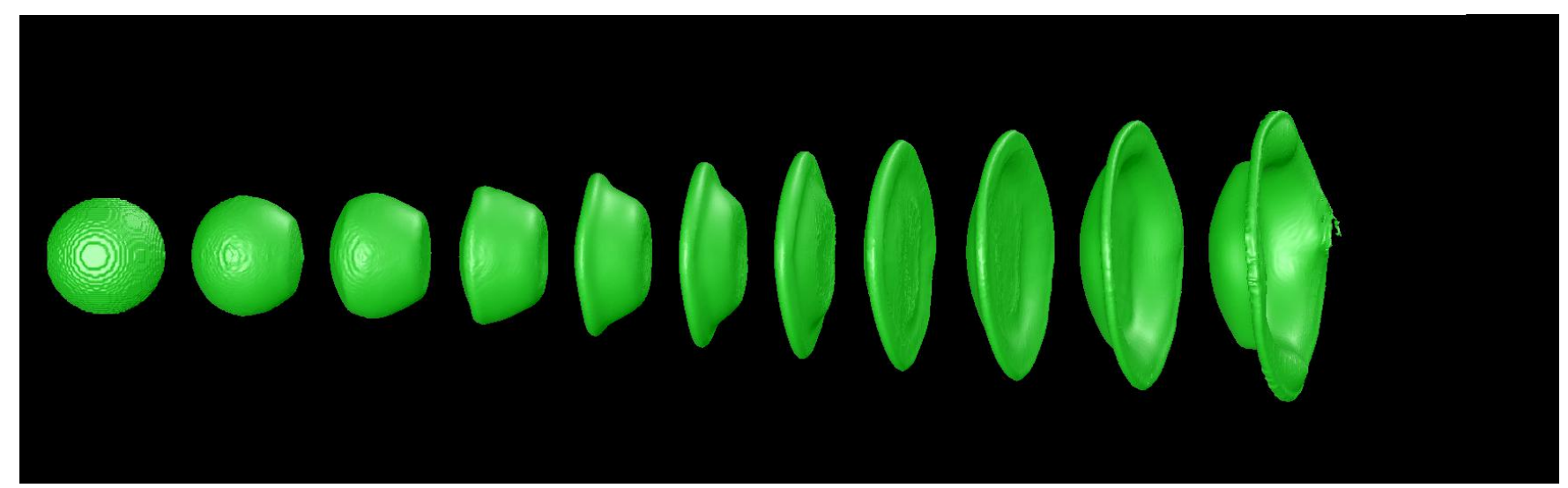

The interval between frames is $0.7 \mathrm{~ms}$.
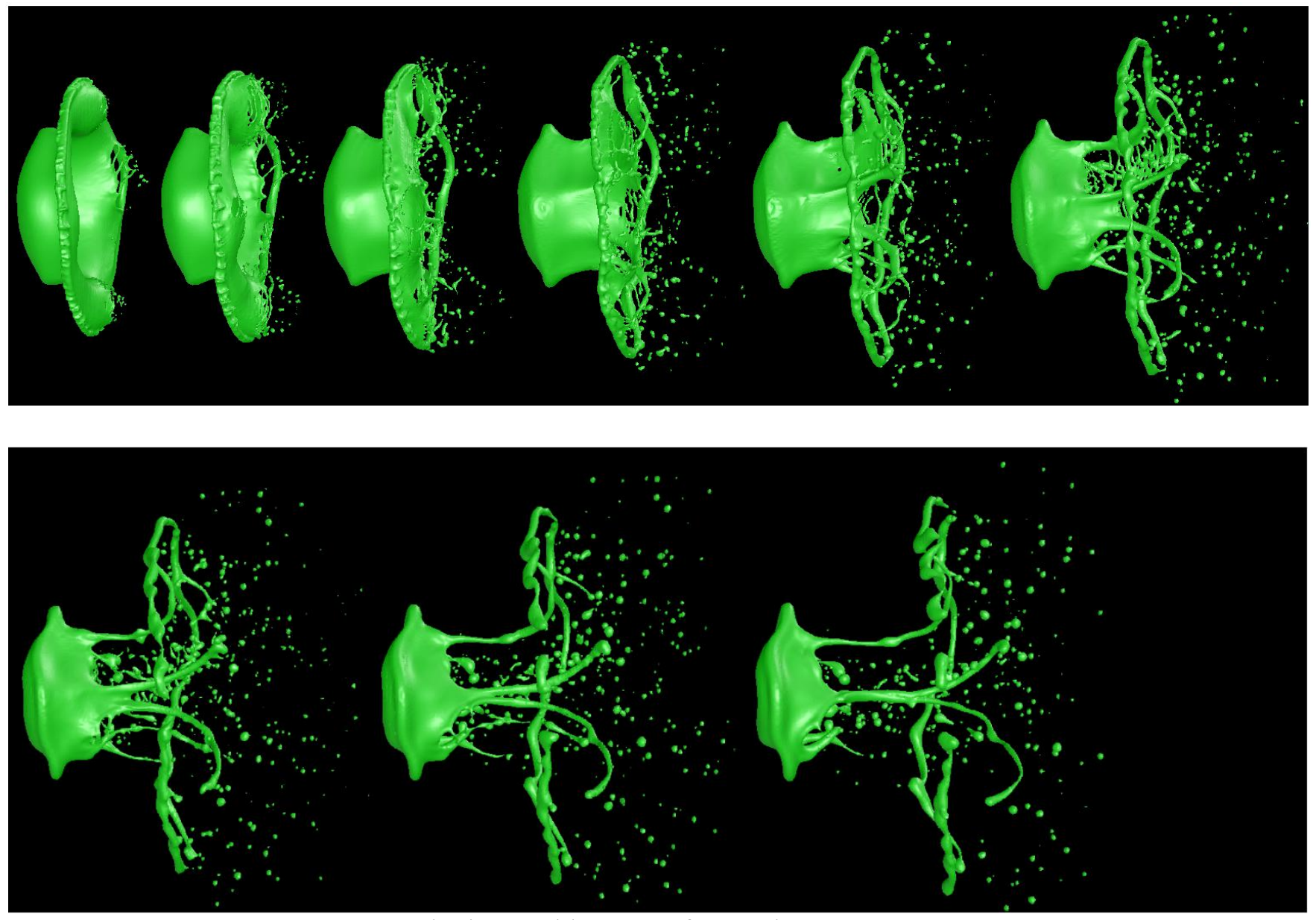

The interval between frames is $0.35 \mathrm{~ms}$.

Fig. 11. Shape of droplet surface at different moments of time at the flow velocity of $25 \mathrm{~m} / \mathrm{s}$. 

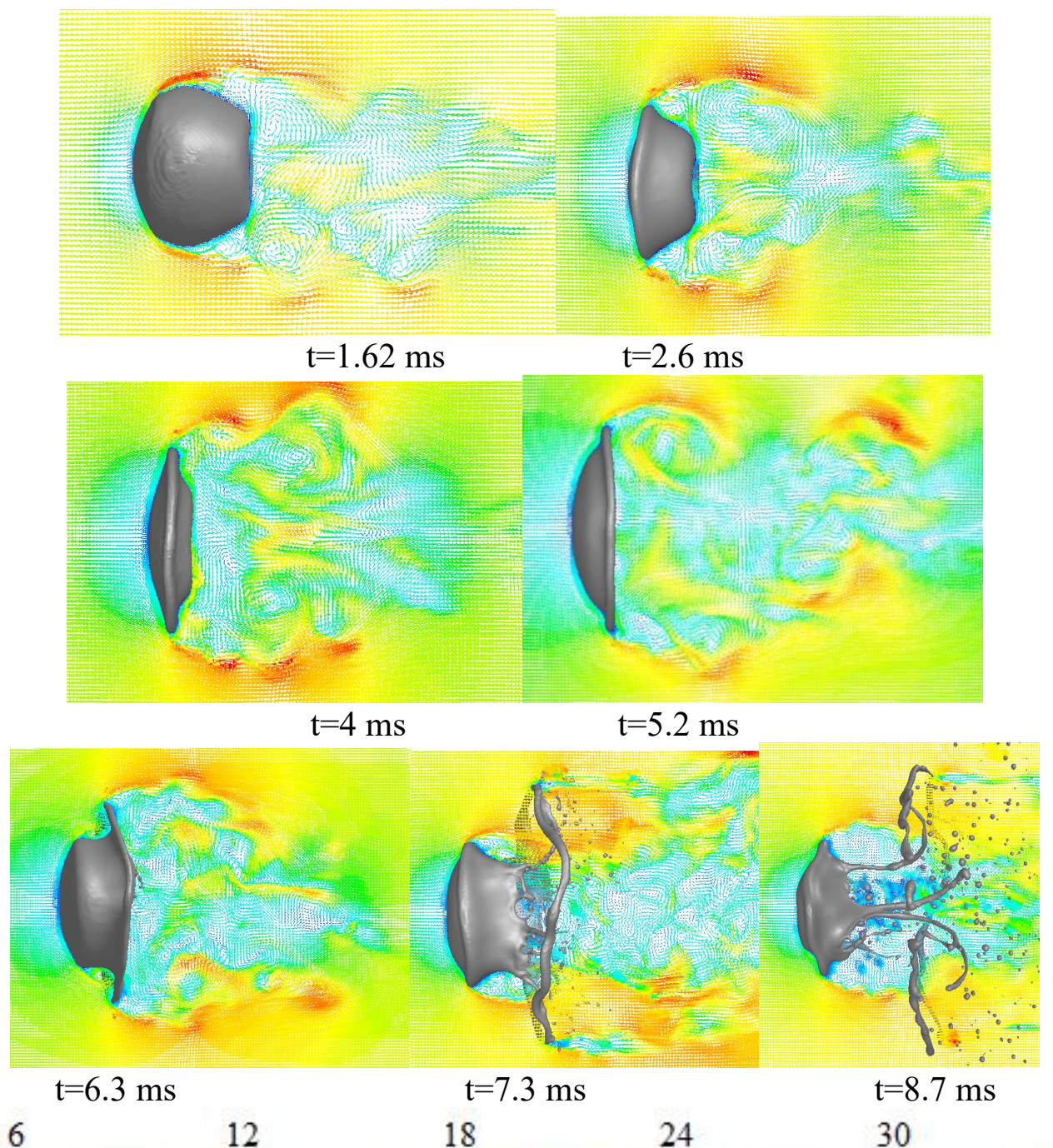

Fig. 12.Snapshot of the stream flowing around a droplet at different moments of time at the flow velocity of $25 \mathrm{~m} / \mathrm{s}$.

Fig. 13 depicts the evolution of the droplet behavior at the flow velocity of $35 \mathrm{~m} / \mathrm{s}$. This corresponds to the Weber number of 104. The droplet behavior with this Weber number differs from the afore described cases. At the initial stage, the droplet - as in the previous cases - deforms into a thinning film. Then, small droplets blow off along the whole external surface of this film, which is rapidly shrinking. For droplets of uniform liquids, this mode is referred to as sheet stripping or sheet-thinning breakup in literature. 


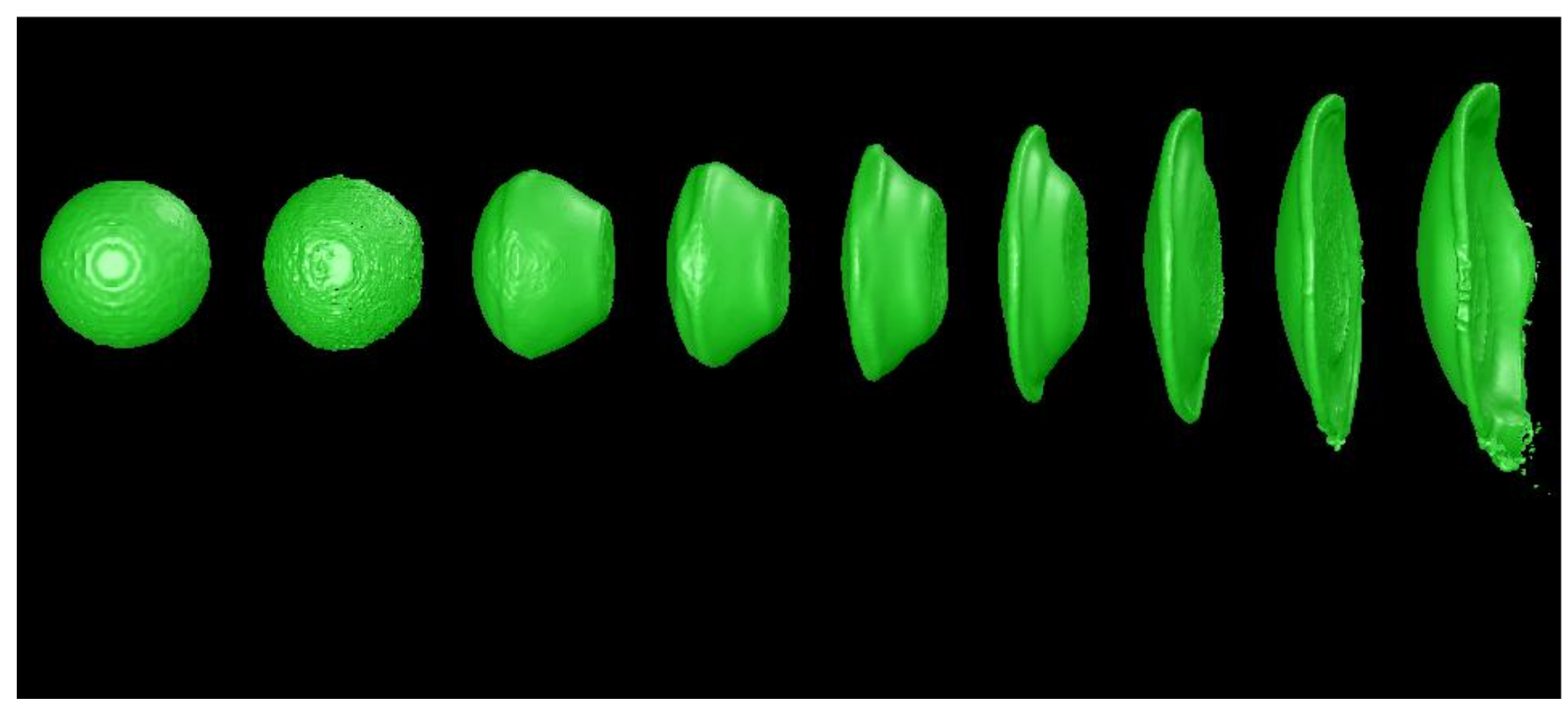

The interval between frames is $0.4 \mathrm{~ms}$.
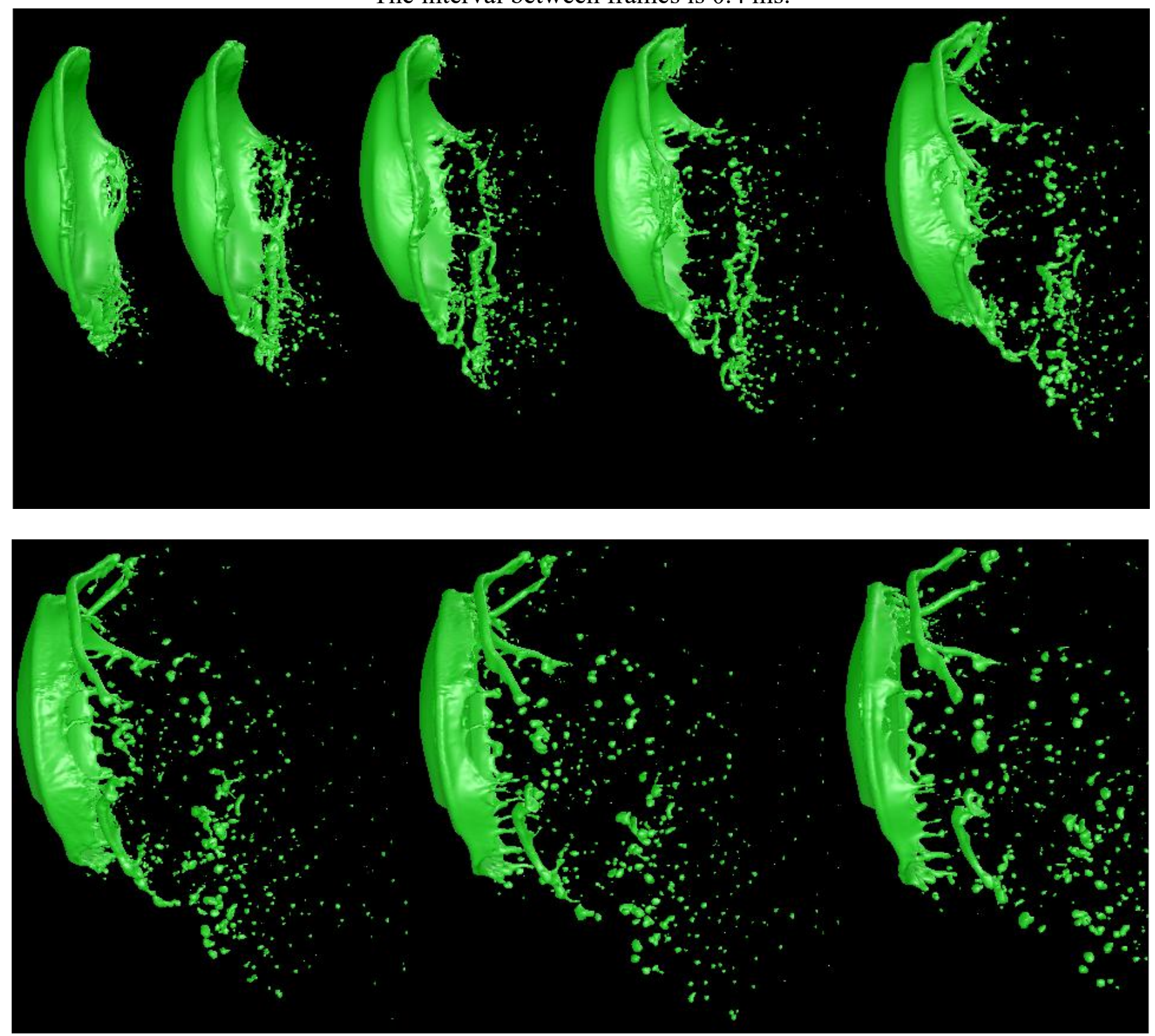

The interval between frames is $0.2 \mathrm{~ms}$.

Fig. 13. Shape of droplet surface at different moments of time at the flow velocity of $35 \mathrm{~m} / \mathrm{s}$. 
The vortex-like structure of the flow behind the droplets in this mode is depicted in Fig. 14. The vortex-like structure is presented as Q-criterion isosurfaces colored by the flow velocity modulus. As mentioned before, the stream behind the droplet is intricate with the formation of both longitudinal and circular vortices.

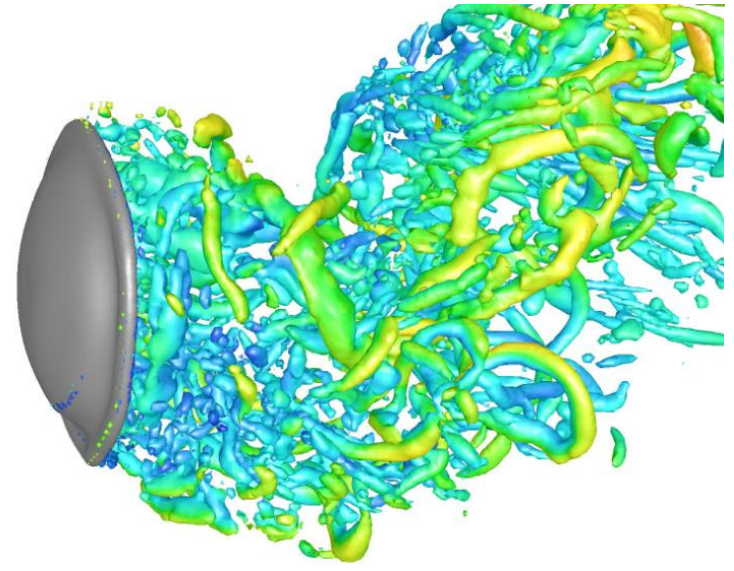

$2 \mathrm{~ms}$

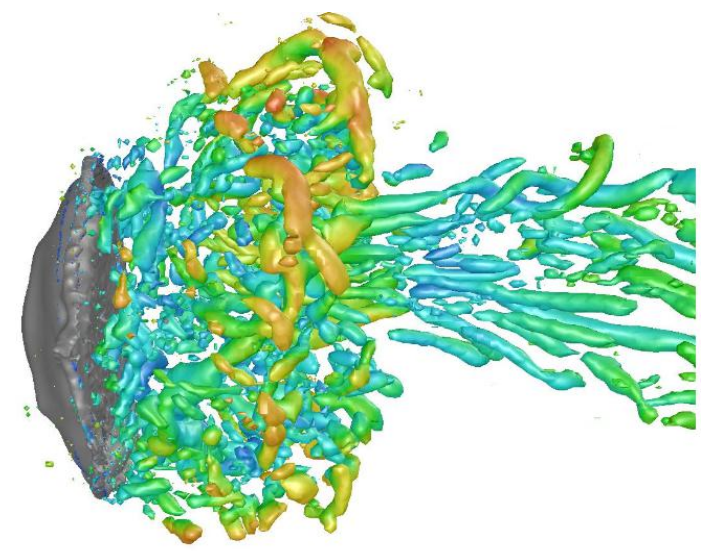

$2.8 \mathrm{~ms}$

Fig. 14. The vortex-like structure of the stream behind the droplet at different moments of time at the flow velocity of $35 \mathrm{~m} / \mathrm{s}$.

At the flow velocity of over $45 \mathrm{~m} / \mathrm{s}(\mathrm{We}=172)$, the so-called catastrophic droplet breakup mode occurs. The catastrophic breakup is associated with the formation of long waves on the droplet surface. Several large fragments break away from these waves and split repeatedly into smaller droplets. Simultaneously, droplets themselves break away from wave crests on the droplet surface. The behavior of a droplet in this mode for the flow velocity of $50 \mathrm{~m} / \mathrm{s}(\mathrm{We}=212)$ is depicted in Fig. 15. Clearly, at such velocities, a droplet quickly turns into a cloud of fine droplets. 

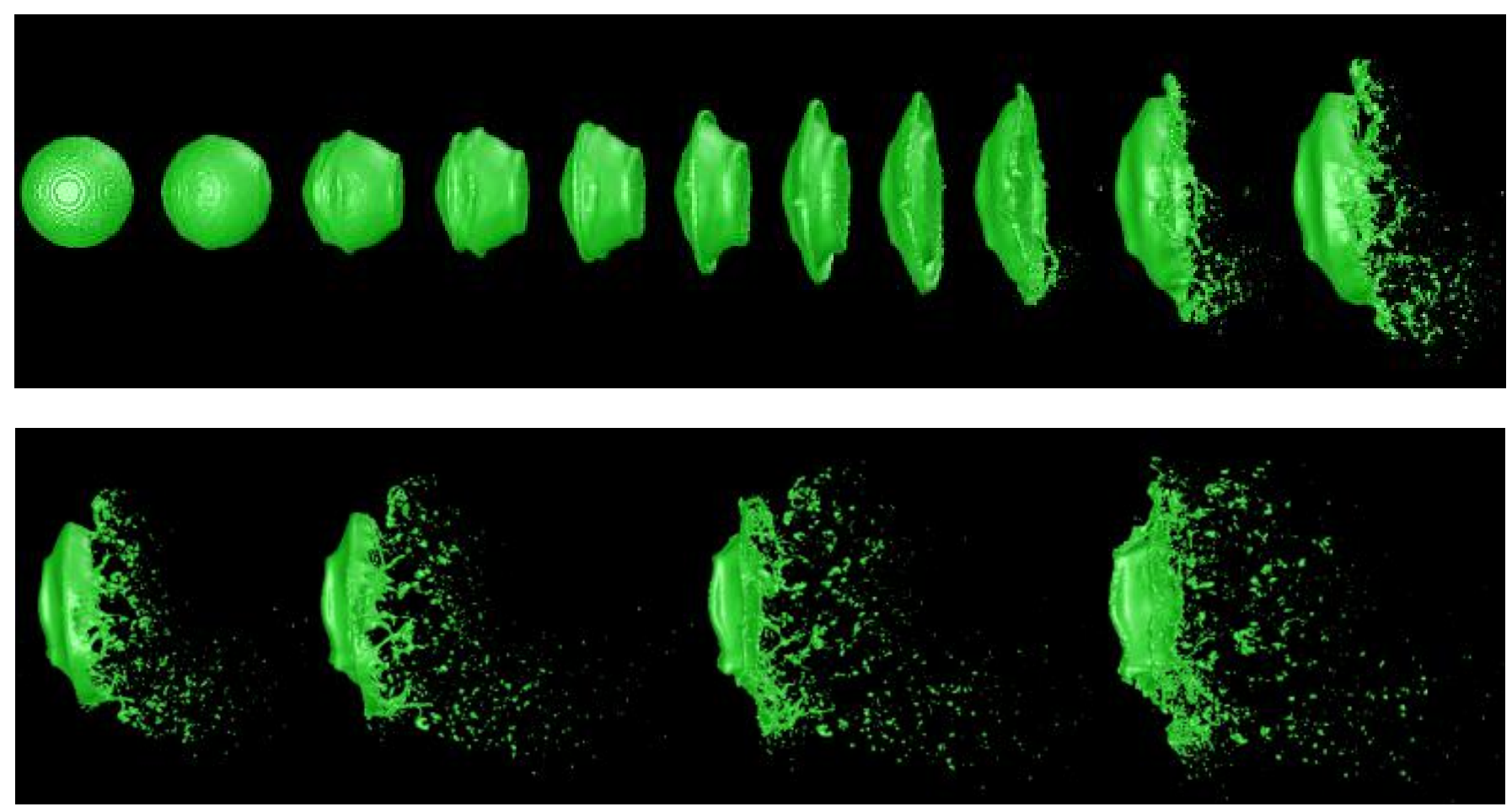

Fig. 15. Shape of droplet surface at different moments of time at the flow velocity of $50 \mathrm{~m} / \mathrm{s}$. The interval between frames is $0.2 \mathrm{~ms}$.

The analysis of computation results distinguishes approximate boundaries of the breakup modes of a CWSP droplet under study. These boundaries are presented in Table 3.

Table 3. Secondary breakup modes of CWSP droplets

\begin{tabular}{|c|c|c|c|}
\hline No breakup & $\begin{array}{c}\text { Bag and multi-mode } \\
\text { breakup }\end{array}$ & $\begin{array}{c}\text { Shear breakup } \\
\text { Catastrophic } \\
\text { breakup }\end{array}$ \\
\hline $\mathrm{We}<10$ & $12<\mathrm{We}<100$ & $\mathrm{We}>100$ & $\mathrm{We}>180$ \\
\hline
\end{tabular}

During the entire breakup process, the initial spherical drop is strongly deformed by the air jet flow. Conventionally, the relation of the current lateral dimension of a droplet to the initial diameter $\mathrm{D} / \mathrm{D}_{0}$ is taken as the quantitative characteristic of droplet breakup modes. This relation for different flow velocities is shown in Fig. 16. Here $\mathrm{D}_{0}$ is the diameter of the initial drop and $\mathrm{D}$ is the cross-stream diameter of the deforming drop. Figure 16 shows the temporal evolution of $\mathrm{D} / \mathrm{D}_{0}$ from dimensionless $\mathrm{t} / \mathrm{T}$.

Where $T=\frac{D_{0}\left(\rho_{l} / \rho_{g}\right)^{1 / 2}}{U_{g}}$,

here $\rho_{g}, \rho_{l}$ and is the density of the surrounding gas and liquid of droplet, $\mathrm{U}_{\mathrm{g}}$ is gas velocity.

Chou and Faeth [30] showed that the temporal evolution of $\mathrm{D} / \mathrm{D}_{0}$ in bag breakup regime is

$$
D / D_{0}=1+0.5 \cdot\left(\frac{t}{T}\right) .
$$


For bag-stamen droplet breakup regime Hui Zhao et al [31] proposed formulas:

$$
D / D_{0}=1+0.54 \cdot\left(\frac{t}{T}\right)^{1.67} \text {. }
$$

These correlations were obtained in experiments for drops of water, ethanol and glycerol. We also show them in Figure 16. As can be seen, they are in agreement with our results for bag breakup regime.

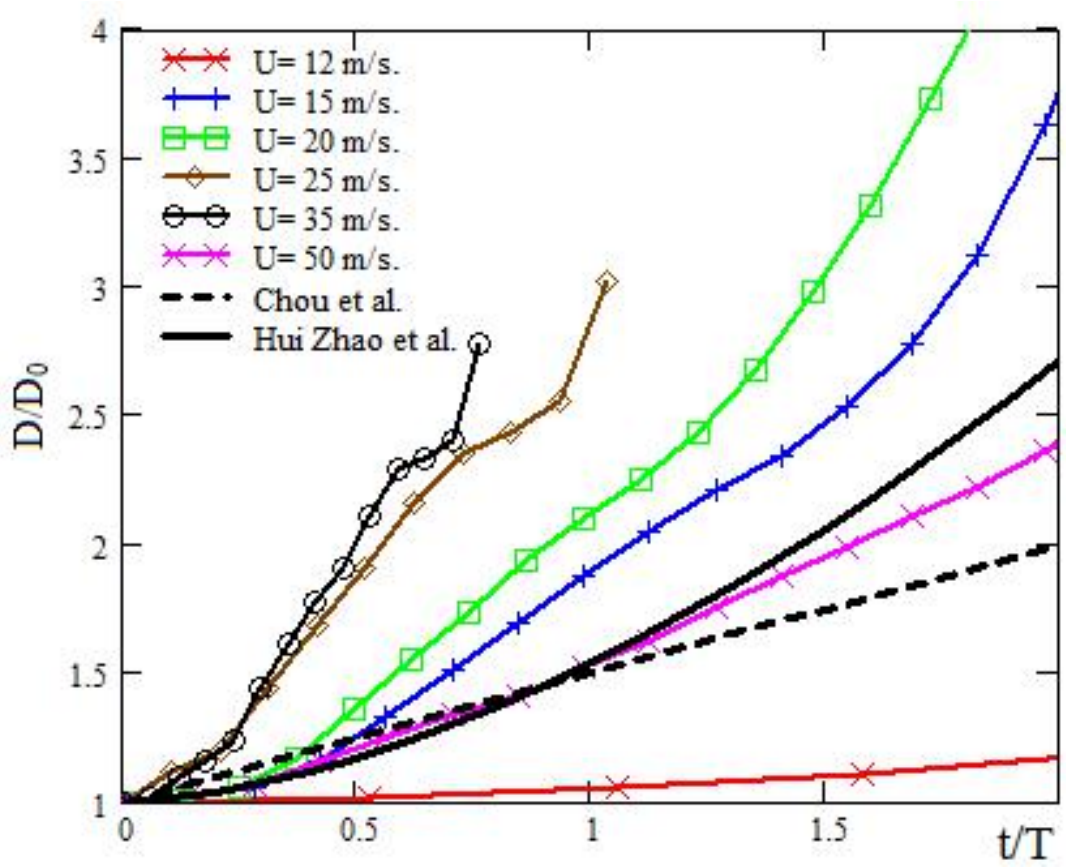

Fig. 16. Lateral dimension of a droplet versus dimensionless time.

The most important characteristic of droplet breakup is its maximum dimension before breakup, Dmax. After a droplet reaches this size, separate fragments start to tear off. The dependence of the relation Dmax/D for the CWSP under study is presented in Fig. 17. Evidently, the relation Dmax/D depends on the Weber number. With an increase in the Weber number, the relation Dmax/D decreases. For reference, Fig. 17 also displays experimental data from [32] for water droplets and from [10] for CWS droplets with the following properties: $\rho=1166 \mathrm{~kg} / \mathrm{m}^{3}$ and $\mu=0.576 \mathrm{~Pa} \times \mathrm{s}$. Clearly, our calculations are in overall qualitative agreement with the experiments. 


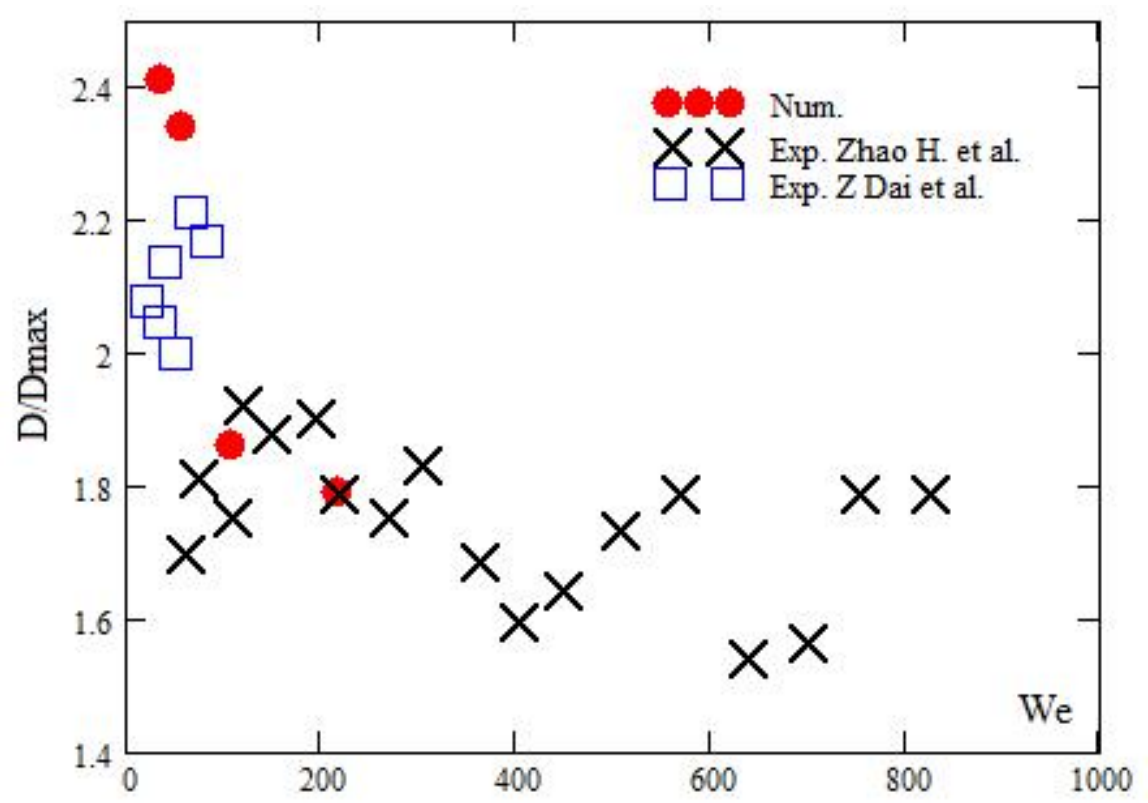

Fig. 17. Dimensionless time until droplet breakup versus We number for filter cake $\mathrm{C}(50 \%)$.

One more characteristic of the secondary breakup is the time it takes a droplet to reach the maximum lateral size Dmax followed by droplet breakup. For this purpose, we introduce the dimensionless initial time, which is determined from the following expression:

$$
T_{\max }=\frac{U_{g} * t_{\max }}{D_{0}\left(\rho_{l} / \rho_{g}\right)^{1 / 2}}
$$

The dependence of dimensionless time until droplet breakup on We number for filter cake $\mathrm{C}(50 \%)$ is shown in Fig. 18. For comparison, Fig. 18 also contains the experimental data from [32] for water droplets and the experimental correlation from [10] for CWS droplets. According to this correlation:

$\mathrm{T}_{\max }=1.9(\mathrm{We}-12)^{-0.25}\left(1+4.5 \mathrm{Oh}^{0.45}\right)$, where $\mathrm{Oh}$ is the Ohnesorge number. 


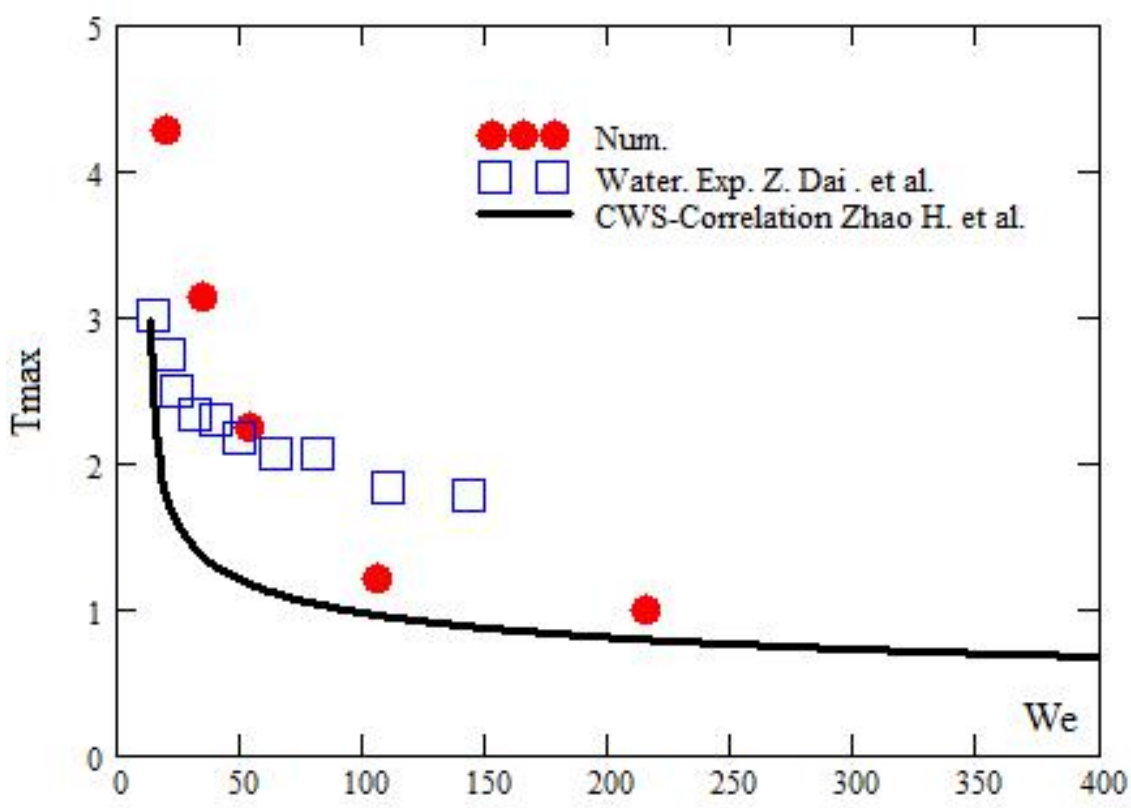

Fig. 18.Dimensionless time of breakup initiation versus We number for filter cake $\mathrm{C}$ (50\%).

Clearly, for large Weber numbers, this correlation adequately describes our computation results. This correlation was obtained for CWS droplet breakup at We $>200$.

\section{Conclusion}

The secondary breakup of isolated CWSP droplets was modeled numerically. We established the droplet breakup modes depending on the Weber number ranging from 7 to 212 . The structure of the stream behind droplets was studied in detail and the numerical method was tested. The results are in good agreement with the results of known experiments. As a result, the research has established the dependence of the Dmax/D change and dimensionless time of droplet breakup initiation on the Weber number.

The work is performed at partial financial support of the Ministry of Education and Science of the Russian Federation within the state assignment with Siberian Federal University in 2018 (No. 16.8368.2018. and Russian Science Foundation (the project No. 15-19-10003).

\section{References}

[1] Tsepenok A.I., OvchinnikovYu.V., StrizhkoYu.V., Lutsenko S.V. Combustion of man-made composite liquid fuel in cyclone precombustor.Energetik 2011;.No. 7. P. 45-47.

[2] Xu M., Liu H-F., Zhao H., Li W. Effect of oily sludge on the rheological characteristics of coke-water slurry. Fuel.2014; 116. P. 261-266.

[3] M. Kurmangaliev and V. Nekrasov. Breakup of water-coal slurry drops in air flows. Fluid Mech. Sov.Res. 1975; 4. 4. 
[4] T. U. Yu, S. W. Kang, and J. M. Beer. Secondary atomization of coal water fuels for gas turbine applications.MIT Energy Lab Report. 1988; No. 88- 008

[5] M. Sitarski.On the feasibility of secondary atomization of small slurry droplets exposed to intense thermal radiation.Combust.Sci. Technol.1987; 54, 177.

[6] M. Sitarski, Thermal dynamics of a small vaporizing slurry droplet in a hot and radiant environment feasibility of the secondary atomization, Combust. Sci. Technol.1990; 71, 53.

[7] Hui Zhao, Hai-Feng Liu, Jian-Liang Xu, and Wei-Feng Li. Secondary breakup of coal water slurry drops. Phys. Fluids. 2011. 23, 113101; doi: 10.1063/1.3659495.

[8] Tavangar S., Hashemabadi S.H., Saberimoghadam A. CFD simulation for secondary breakup of coalwater slurry drops using OpenFOAM. Fuel Processing Technology, 2015; 132.P.153-163.

[9] Zhao H., Liu H-F., Xu J-L., Li W.F., Cheng W. Breakup and atomization of a round coal water slurry jet by an annular air jet. Chemical Engineering Science. 2012. V. 78. P. 63-74.

[10] Zhao H., Hou Y., Liu H., Tian X., Xu J., Li W., Liu Y., Wu F., Zhang J., Lin K. Influence of rheological properties on air-blast atomization of coal water slurry. J. Non-Newtonian Fluid Mech. 2014. 211. P. 1-15.

[11] Aggarwal S.K., Peng F. A review of droplet dynamics and vaporization modeling for engineering calculations.J. Eng. Gas Turbines Power.1995. 117. P. 453-461.

[12] Minakov A.V. Numerical algorithm for moving boundary fluid dynamics problems and its testing.Computational Mathematics and Mathematical Physics. 2014. Vol. 54, N 10. P. 1560-1570.

[13] Frank A.M. Discrete models of incompressible liquid.M. Fizmatlit. 2001. P. 206.

[14] Burago N.G. Numerical solution of problems of continuum mechanics with movable interfaces.Doctoral dissertation. Moscow. 2003. P. 222.

[15] Kothe D.B., Rider W.J. Volume tracking of interfaces having surface tension in two and three dimensions. S.J. AIAA. 1996; Paper - 96.

[16] Osher S., Sethian J.A. Fronts propagating with curvature-dependent speed: algorithms based on Hamilton-Jacobi formulations. Journal of computational physics.1988; Vol. 79. P. 12-49.

[17] Afanasyev K.E., Ilyasov A.E., Makarchuk R.S., Popov A.Yu. Numerical simulation of liquid streams with free boundaries by SPH and MPS .Computation technologies.2006; V. 11.Special issue. P. 26-44.

[18] Hansbo P.A. Free-lagrange finite element method using space-time elements. // Comp. meth. appl. Mech. Engrg. 2000; Vol. 188. P.347-361.

[19] Harlow F.H., Welch J.E. Numerical calculation of time-dependent viscous incompressible flow of fluid with free surface.The physics of fluids.1965; Vol. 8, №. 12. P.2182-2189.

[20] Hirt C.W, Nichols B.D. Volume of fluid (VOF) method for the dynamics of free boundaries.Journal of computational physics.1981; No. 39. P. 201-226. 
[21] Minakov A.V., Pervukhin M.V., Platonov D.V., Khatsayuk M.Y.Mathematical model and numerical simulation of aluminum casting and solidification in magnetic fields with allowance for free surface dynamics. Computational Mathematics and Mathematical Physics.2015; Vol. 55, 12. P. 2066-2079.

[22] Shebeleva A.A., Minakov A.V., Yagodnitsina A.A., Andyuseva V.G. Numerical simulation of twocomponent flow fluid - fluid in the microchannel T- type.MATEC Web of Conferences, Heat and Mass Transfer in the Thermal Control System of Technical and Technological Energy Equipment, 2015.V 23, N 01043.

[23] Gavrilov A.A., Minakov A.V., Dekterev A.A., RudyakV.Ya. Numerical algorithm for modeling settled laminar streams of non-Newtonian liquids in annular gap with eccentricity.Computation technologies.2012; V. 17.No. 1.P. 44-56.

[24] A. Gavrilov, V. Rudyak. Reynolds-averaged modeling of turbulent flows of power-law fluid. Journal of Non-Newtonian Fluid Mechanics 227 (2016) 45-55.

[25] Brackbill J.U., Kothe D.B., Zemach C.A. Continuum method for modeling surface tension. J. Comput. Phys. 1992; Vol. 100.P. 335- 54.

[26] J. Smagorinsky. "General Circulation Experiments with the Primitive Equations.I. The Basic Experiment". Month.Wea. Rev.1963; 91.P. 99-164.

[27] Minakov A.V., Rudyak V.Y., Gavrilov A.A., Dekterev A.A. Mixing in a T-shaped micromixer at moderate Reynolds numbers. Thermophys.Aeromech. 2012; 19, P. 385-395.

[28] Minakov A.V., Rudyak V., Dekterev A., Gavrilov A. Investigation of slip boundary conditions in the T-shaped microchannel J. Heat Fluid Flow. 2013; 43, P. 161-169.

[29] Glushkov D.O., Strizhak P.A. Ignition of composite liquid fuel droplets based on coal and oil processing waste by heated air flowюю Journal of Cleaner Production. 2017; V. 165. P. 1445-1461.

[30] W. H. Chou and G. M. Faeth. Temporal properties of secondary drop breakup in the bag breakup regime. Int. J. Multiphase Flow 24, 889 (1998).

[31] Hui Zhao, Hai-Feng Liu, Jian-Liang Xu, Wei-Feng Li, and Kuang-Fei Lin. Temporal properties of secondary drop breakup in the bag-stamen breakup regime. Phys. Fluids 25, 054102 (2013); doi: 10.1063/1.4803154

[32] J.Z. Dai, G.M. Faeth. Temporal properties of secondary drop breakup in the multimode breakup regime. International Journal of Multiphase Flow. 2001; 27, P 217-236. 